\title{
Care coordination and provider stress in primary care management of high-risk patients
}

\author{
Adeyemi Okunogbe, MB.ChB, MSc ${ }^{1,2}$, Lisa S. Meredith, PhD 1,2,3, Evelyn T. Chang, MD, MSHS ${ }^{3,4,5}$, \\ Alissa Simon, $M A^{3}$, Susan E. Stockdale, PhD ${ }^{3,5}$, and Lisa V. Rubenstein, MD, MSPH ${ }^{1,6}$ \\ 'RAND Corporation, Santa Monica, CA, USA; ${ }^{2}$ Pardee RAND Graduate School, Santa Monica, CA, USA; ${ }^{3}$ VA HSR\&D Center for Healthcare

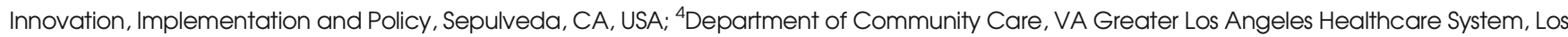 \\ Angeles, CA, USA; ${ }^{5}$ Department of Psychiatry and Bio-behavioral Sciences, University of California, Los Angeles, CA, USA; ${ }^{6}$ Department of \\ Medicine, University of California at Los Angeles, Los Angeles, CA, USA.
}

\begin{abstract}
BACKGROUND: Care coordination is a critical component of managing high-risk patients, who tend to have complex and multiple medical and psychosocial problems and are typically at high risk for increased hospitalization and incur high health care expenditures. Primary care models such as the patient-centered medical homes (PCMHs) are designed to improve care coordination and reduce care fragmentation. However, little is known about how the burden of care coordination for high-risk patients influences PCMH team members' stress.
\end{abstract}

OBJECTIVE: To evaluate the relationship between provider stress and care coordination time in high-risk patient care and whether availability of help is associated with reduced stress.

STUDY DESIGN: Multivariable regression analysis of a cross-sectional survey of PCMH primary care providers (PCPs) and nurses.

PARTICIPANTS: A total of 164 PCPs and 272 nurses in primary care practices at five geographically diverse Veteran Health Administration (VA) medical center health systems. MAIN MEASURES: The main outcome variable was provider stress due to high-risk patient care. Independent variables were the reported proportion of high-risk patients in $\mathrm{PCP} /$ nurse patient panels, time spent coordinating care for these patients, and provider satisfaction with help received in caring for them.

KEY RESULTS: The response rate was 44\%. Spending more than $8 \mathrm{~h}$ per week coordinating care was significantly associated with a 0.21 -point increase in reported provider stress compared to spending $8 \mathrm{~h}$ or less per week (95\% CI: 0.04-0.39; $p=0.015$ ). The magnitude of the association between stress and care coordination time was diminished when provider satisfaction with help received was included in the model.

CONCLUSIONS: Perceived provider stress from care of high-risk patients may arise from challenges related to coordinating their care. Our findings suggest that the perception of receiving help for high-risk patient care may be valuable in reducing provider stress.

Electronic supplementary material The online version of this article (https://doi.org/10.1007/s11606-017-4186-8) contains supplementary material, which is available to authorized users.

Received December 2, 2016

Revised May 11, 2017

Accepted September 6, 2017

Published online October 2, 2017
KEY WORDS: Care coordination; High-risk patients; Provider stress; Patient-centered medical home model; Primary care.

J Gen Intern Med 33(1):65-71

DOI: $10.1007 / \mathrm{s} 11606-017-4186-8$

(C) Society of General Internal Medicine 2017

\section{INTRODUCTION}

Care coordination is a critical component of managing highrisk patients (HRPs) due to the number and diversity of their needs, which may require care from as many as 16 physicians in a year. ${ }^{1}$ These individuals tend to have complex and multiple medical and psychosocial problems and are typically at a high risk of increased hospitalizations and poor health outcomes and incur high health care expenditures. ${ }^{2-5}$ Some research has suggested that care coordination in primary care settings can be challenging ${ }^{6-10}$ and may overstress primary care providers (PCPs). ${ }^{1}$ As the number of Americans with multiple chronic conditions increases, care coordination could represent a significant burden for providers.

Recent research shows that the level of stress among medical professionals continues to rise across the US. Nationally, in $2014,54 \%$ of physicians reported at least one symptom of burnout, representing an increase of about 9 percentage points from 2011 estimates. ${ }^{11}$ Provider burnout has known negative effects, including worsened mental and physical health, ${ }^{12}$ reduced work performance, ${ }^{13}$ greater patient safety risks, ${ }^{14}$ lower organizational commitment, ${ }^{15,16}$ and increased workforce turnover and demand. ${ }^{17}$

Defined as "the deliberate organization of patient care activities between two or more participants (including the patient) involved in a patient's care to facilitate the appropriate delivery of health care services," ${ }^{18-21}$ care coordination is positively associated with patient satisfaction outcomes. ${ }^{22}$ Some of the available, although limited research suggests that better care coordination between referring physicians and specialists increases physician satisfaction with specialty care in a pediatric primary care network. ${ }^{23}$ However, little is known about how the burden of care coordination for HRPs affects provider stress. In this article, we explore care coordination of 
HRPs from the perspective of providers and its relationship with stress.

The context of our study is the Veterans Health Administration (VHA) patient-centered medical home (PCMH) model. VHA's PCMH model, implemented in 2010, is a team-based model of primary care that is patient-centered, comprehensive, coordinated, and accessible and that employs a systems-based approach to quality and safety. ${ }^{24}$ While some elements of team-based care such as participatory decision-making have been found to be associated with lower burnout, ${ }^{25-29}$ other recent evidence suggests that transformation of primary care due to adoption and implementation of the PCMH model in the VHA may be associated with emotional exhaustion among providers. ${ }^{26}$ Our study focuses on a critical component of the PCMH model — care coordination — and assesses the degree to which time spent coordinating care of HRPs in this setting is associated with provider stress.

As shown in Fig. 1, we hypothesized that (1) the perceived proportion of HRPs is positively associated with time spent on care coordination, (2) time spent on care coordination is negatively associated with provider satisfaction with help received for caring for HRPs, (3) proportion of HRPs is positively associated with provider stress, (4) time spent on care coordination is positively associated with provider stress, and (5) provider satisfaction with help received for caring for HRPs is negatively associated with stress. We test these hypotheses with survey data from VHA PCPs and nurses.

To our knowledge, no study exists that investigates the association between the care coordination burden and reported stress among PCPs and nurses caring for HRPs. This study therefore fills a critical gap in the available evidence relating to care coordination in two ways. First, it sheds light on care coordination, an essential component of the PCMH model, and its association with provider stress in HRP management. Second, it provides potential evidence that certain mechanisms may alleviate and counter the stress experienced by providers because of the increased care coordination demands from caring for HRPs.

\section{METHODS}

\section{Design, setting and sample}

The survey was conducted as part of the evaluation of a national pilot project to provide enhanced primary care for HRPs at five VHA healthcare systems (in Ohio, Georgia, North Carolina, Wisconsin, and California) and was developed in collaboration with the RAND Corporation.

All PCPs (physicians, MDs/DOs; nurse practitioners, NPs; physician assistants, PAs) and nurses (registered nurses, RNs; licensed practical nurses, LPNs; licensed vocational nursesLVNs) at these five healthcare systems $(n=1000)$ were invited to participate in an online survey about their experiences providing team-based care to HRPs. The survey was fielded from December 3, 2014 through January 31, 2015 and was extended from April 10, 2015 through May 29, 2015 with a paper-and-pen version to improve the response rate. The survey was administered using REDCap. This study was part of a quality improvement program evaluation targeting HRPs that constituted a VHA health care operations activity rather than research and did not require Institutional Review Board (IRB) approval or exemption.

\section{Measures}

Primary outcome variable. Our main outcome measure was the reported stress of providers in caring for HRPs. This was a multi-item scale derived by averaging across 4 items that measure provider perception of stress from caring for their HRPs (see Online Supplemental Table 1). Items were rated on a 5-point Likert agreement scale, and the averaged scale had high reliability (Cronbach's alpha $=0.78$ ) with a higher score indicating greater stress from caring for HRPs. The scale has not been previously validated. However, the items had face validity with a sample of PCPs/nurses who pretested the items, and exploratory factor analysis found that these items measured a similar underlying construct and loaded on the same factor.

Independent variables. Key independent variables included proportion of HRPs in the patient panel, care coordination time, and provider satisfaction with the help received to care for HRPs. The proportion of HRPs in the patient panel was a three-category variable: $0-10 \%, 11-20 \%$, and $>20 \%$ (Table 1).

We derived care coordination time from responses to the question "How much time during a typical week (outside of the patient visit) do you typically spend coordinating care for your patients?" Response categories were $0-4 \mathrm{~h}, 5-8 \mathrm{~h}, 9$ $20 \mathrm{~h}, 21-30 \mathrm{~h}$, and more than $30 \mathrm{~h}$ (per week). For this analysis, we created a binary variable: $\leq 8 \mathrm{~h}$ vs. $>8 \mathrm{~h}$ per week based on the distribution of responses (Table 1).

Provider satisfaction with the help they receive to care for HRPs ("Overall, I am satisfied with the help I receive to care for my high-risk patients") was measured on a 5-point single Likert-type agreement item where higher scores indicate greater satisfaction with help received.

Covariates. We controlled for demographic characteristics [gender (female vs. male), age group (45 years or older, 3645 years, and 26-35 years of age)] and practice characteristics [provider type (nurse vs. PCPs), staffing of PACT team (full vs. partial), and years of practice at the current VHA clinic].

\section{Statistical analysis}

We conducted univariate and bivariate analyses to describe the respondents' characteristics and the relationships among independent variables. We also employed multivariate regression 


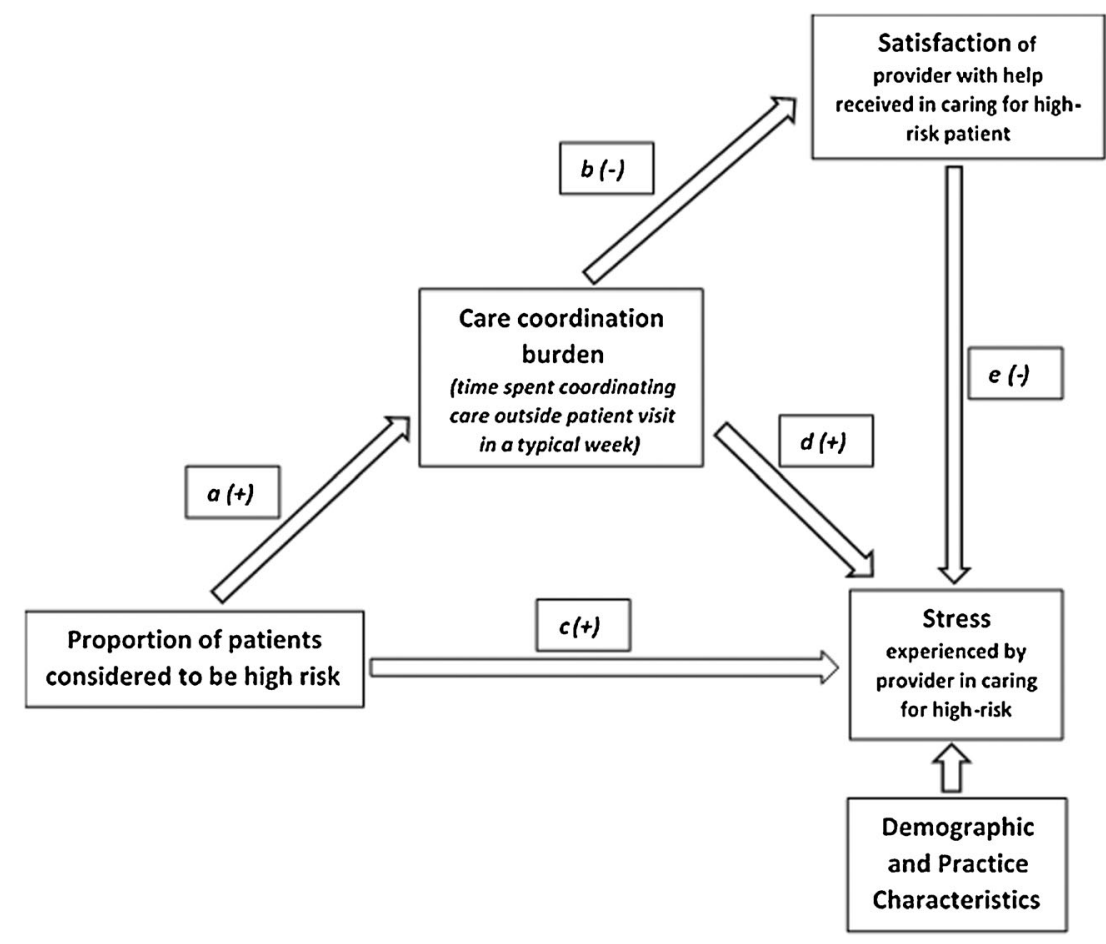

Fig. 1 Hypothetical framework for care coordination and provider stress in high-risk patient management

to estimate the associations for each of the pathways in our hypothetical framework (Fig. 1). First, we explored the association between the proportion of HRPs in a provider's patient panel and care coordination time. We hypothesized that this relationship is positive (denoted as $a$ ). Second, we hypothesized that care coordination time is negatively associated with provider satisfaction with the help received in caring for their HRPs (denoted as $b$ ). Lastly, we explored the association between the main outcome variable (i.e., provider stress in caring for HRPs) and independent variables - proportion of HRPs (denoted as c), care coordination time (denoted as $d$ ), and providers' satisfaction with the help received in caring for their HRPs (denoted as $e$ ). We hypothesized that their associations with stress were positive for both proportion of HRPs and care coordination time and negative for providers' satisfaction with help received.

We tested these hypotheses using three sets of models. For each set of models, we sequentially added variables starting with the main explanatory variables, followed by demographic characteristics and finally professional/practice characteristics. We chose this sequential modeling approach to better understand the unique contributions of each group of explanatory variables. We included medical center fixed effects in our regressions and used robust standard errors. We used logistic, ordered logistic, and linear regression models as appropriate for the outcome variable being predicted.

All analyses were performed using standard statistical software (Stata 11.2) and included post-stratification weights to adjust for survey non-response by provider type, provider gender, medical center location, and clinical team. Weights were calculated based on the sampling frame of all PCPs and nurses in the five medical centers. We conducted multiple imputation using five replications for all missing data (missingness rate of $8 \%$ ) and conducted analyses on both imputed and non-imputed data sets.

\section{RESULTS}

Sample characteristics. A total of 436/1000 PCPs and nurses completed the survey for a response rate of $44 \%$. Survey respondents were significantly different from nonrespondents with respect to gender, job type, and location of medical center. More females responded compared to nonrespondents, one medical center had more respondents compared to non-respondents, and fewer proportions of both nurses and PCPs responded compared to non-respondents. The majority of respondents were female $(78.4 \%)$ and $>$ 45 years in age $(68.7 \%)$ (Table 1). On average, survey respondents had 19 years of clinical experience and had been working in their current VHA clinic for about 7 years. We found no statistically significant differences in years of clinical or current VHA work experience between PCPs and nurses. Table 1 also shows the summary statistics for the main dependent variable and the three main independent variables. PCPs and nurses did not differ significantly in the proportion of HRPs reported. However, nurses were significantly more likely to report less stress in caring for HRPs $(p<0.001)$, were more likely to spend more time coordinating care $(p=0.01)$, and were more satisfied $(p=0.001)$ with the help they receive to care for HRPs than PCPs. 
Table 1 Descriptive Statistics of Primary Care Providers (PCPs) and Nurses

\begin{tabular}{|c|c|c|c|c|}
\hline Characteristic & Full sample $(n=436)^{*}$ & Primary care providers $(n=164)^{*}$ & Nurses $(n=272)^{*}$ & $P$ value $^{\dagger}$ \\
\hline \multicolumn{5}{|c|}{ I. Demographic and professional characteristics } \\
\hline Female, n $(\%)$ & $342(78.4)$ & $110(67.1)$ & $232(85.2)$ & $<0.001$ \\
\hline Age range, mode $(\%)$ & & & & 0.21 \\
\hline $26-35$ & $39(9.3)$ & $12(7.5)$ & $27(10.3)$ & \\
\hline $36-45$ & $93(22.1)$ & $30(18.8)$ & $63(24.1)$ & \\
\hline $45+$ & $289(68.7)$ & $118(73.8)$ & $171(65.5)$ & \\
\hline Clinical experience [years], mean (SD) & $18.7(11.1)$ & $18.3(10.3)$ & $19.0(11.6)$ & 0.53 \\
\hline Years in current clinic, mean (SD) & $7.0(6.7)$ & $8.5(8.1)$ & $6.0(5.5)$ & $<0.001$ \\
\hline \multicolumn{5}{|l|}{ Job type, n (\%) } \\
\hline Physician & $117(71.3)$ & $117(71.3)$ & - & $\mathrm{N} / \mathrm{A}$ \\
\hline Nurse practitioner & $43(26.2)$ & $43(26.2)$ & - & $\mathrm{N} / \mathrm{A}$ \\
\hline Physician assistant & $4(2.4)$ & $4(2.4)$ & & $\mathrm{N} / \mathrm{A}$ \\
\hline Registered nurse & $164(60.3)$ & - & $164(60.3)$ & N/A \\
\hline Licensed practical/vocational nurse & $108(39.7)$ & - & $108(39.7)$ & $\mathrm{N} / \mathrm{A}$ \\
\hline Fully staffed team, n (\%) & $291(68.3)$ & $104(35.7)$ & $187(64.3)$ & 0.49 \\
\hline \multicolumn{5}{|c|}{ II. Main dependent and independent variables } \\
\hline Stress, mean (SD)*** & $3.5(0.8)$ & $3.7(0.8)$ & $3.4(0.7)$ & $<0.001$ \\
\hline \multicolumn{4}{|l|}{ Proportion of high-risk patients, $\mathrm{n}(\%)$} & 0.34 \\
\hline $0-10 \%$ & $112(26.4)$ & $49(30.3)$ & $63(24.0)$ & \\
\hline $11-20 \%$ & $143(33.7)$ & $53(32.7)$ & $90(34.2)$ & \\
\hline$>20 \%$ & $170(40.0)$ & $60(37.0)$ & $110(41.8)$ & \\
\hline \multicolumn{4}{|l|}{ Satisfaction with help, n (\%) } & 0.001 \\
\hline Strongly disagree & $30(7.0)$ & $19(11.8)$ & $11(4.2)$ & \\
\hline Disagree & $97(22.8)$ & $45(28.0)$ & $52(19.6)$ & \\
\hline Neither disagree nor agree & $131(30.8)$ & $47(29.2)$ & $84(31.7)$ & \\
\hline Agree & $139(32.6)$ & $46(28.6)$ & $93(35.1)$ & \\
\hline Strongly agree & $29(6.8)$ & $4(2.5)$ & $25(9.4)$ & \\
\hline \multicolumn{4}{|l|}{ Care coordination time weekly, n (\%) } & 0.01 \\
\hline $0-4 \mathrm{~h}$ & $94(21.9)$ & $32(19.8)$ & $62(23.2)$ & \\
\hline $5-8 \mathrm{~h}$ & $106(24.7)$ & $49(30.3)$ & $57(21.4)$ & \\
\hline $9-20 \mathrm{~h}$ & $134(31.2)$ & $59(36.4)$ & $75(28.1)$ & \\
\hline $21-30 \mathrm{~h}$ & $54(12.6)$ & $11(6.8)$ & $43(16.1)$ & \\
\hline$>30 \mathrm{~h}$ & $41(9.6)$ & $11(6.8)$ & $30(11.2)$ & \\
\hline
\end{tabular}

*Number of observations for demographic variables do not sum to full sample sizes because of missing data

$\dagger P$ values of tests for significant differences between PCPs and nurses for characteristics

The full results of our three sets of regression models are presented in Online Supplemental Tables 2-4 and summarized in Table 2. We ran regressions on both imputed and nonimputed data. There were no significant or qualitative differences between results derived from both datasets; hence, we report the non-imputed data results.

Logistic regression findings for care coordination time (pathway $a$ in Fig. 1, Column 1 in Table 2) revealed that reporting 11-20\% HRPs in the patient panel was significantly associated with an increase by a factor of 2.0 in the odds of spending more than $8 \mathrm{~h}$ per week on care coordination compared to having less than $10 \%$ HRPs in a patient panel $(p=0.011)$. Reporting $>20 \%$ HRPs was significantly associated with an increase by a factor of 2.6 in the odds of spending more than $8 \mathrm{~h}$ per week on care coordination compared to having less than $10 \%$ HRPs in a patient panel, $(p=0.001)$.

Ordered logistic regression estimates for $\mathrm{PCP} /$ nurse satisfaction with help received in caring for HRPs (pathway $b$ in Fig. 1, Online Supplemental Table 3) showed that higher care coordination time was associated with lower provider satisfaction $(p<0.001)$ and remained significant after controlling for proportion of HRPs, demographic characteristics, and practice characteristics. In the final model, providers coordinating care for more than $8 \mathrm{~h}$ per week were $46 \%$ less likely to experience a higher level of satisfaction compared to providers spending less than $8 \mathrm{~h}$ per week ( $p=0.002$; Column 2 of Table 2$)$. In addition, PCPs were $61 \%$ less likely to experience a higher level of satisfaction compared to nurses $(p<0.001)$.

For the primary outcome, provider stress from caring for HRPs, we found that having $>20 \%$ HRPs was significantly associated with a 0.23 point increase in reported stress compared to having less than $10 \%$ HRPs in a patient panel (pathway $\mathrm{c}$ in Fig. 1; $p=0.022$; Column 3 in Table 2). Also, having 11-20\% HRPs was associated with a 0.16 point increase in reported stress compared to having less than $10 \%$ HRPs in a patient panel, but not significant $(p=0.117)$. In the final model (Column 5 in Table 2), we found that spending more than $8 \mathrm{~h}$ per week coordinating care was significantly associated with a 0.21 point increase in reported provider stress, holding all other variables constant $(p=0.015)$. This represents a small to medium effect size (Cohen's $d=0.30$ ). In addition, providers that reported more satisfaction with help received in caring for HRPs had lower stress scores (pathway e in Fig. 1; $p=0.001$; Column 5 in Table 2). Our final model (Column 5 in Table 2) explained about one-sixth of the variation in provider stress $(\mathrm{R}$-squared $=0.16)$.

Based on findings from our sets of multivariate regressions, we found evidence suggesting mediation by (1) care coordination time in the relationship between stress and having greater than 20\% HRPs in patient panel compared to having less than 10\% HRPs and (2) provider satisfaction with help received to care for HRPs in the relationship between provider 
Table 2 Adjusted Associations among Care Coordination, Proportion of High-risk Patients, Provider Satisfaction with Help Received, and Provider Stress

\begin{tabular}{|c|c|c|c|c|c|}
\hline & \multicolumn{5}{|l|}{ Outcomes } \\
\hline & \multirow{2}{*}{$\begin{array}{l}\text { Spending more than } 8 \mathrm{~h} / \text { week } \\
\text { in care coordination } \\
\text { Column (1) }\end{array}$} & \multirow{2}{*}{$\begin{array}{l}\begin{array}{l}\text { Provider satisfaction with } \\
\text { help received }^{\S}\end{array} \\
\text { Column (2) }\end{array}$} & \multicolumn{3}{|c|}{ Provider stress } \\
\hline & & & Column (3) & Column (4) & Column (5) \\
\hline \multicolumn{6}{|c|}{ Predictor variables } \\
\hline \multicolumn{6}{|c|}{ Proportion of high-risk patients } \\
\hline $0-10 \%$ & Ref & Ref & Ref & Ref & Ref \\
\hline $11-20 \%$ & $\begin{array}{l}2.04 * \\
(1.18-3.54)\end{array}$ & $\begin{array}{l}1.03 \\
(0.64-1.67)\end{array}$ & $\begin{array}{l}0.16 \\
(-0.04-0.36)\end{array}$ & $\begin{array}{l}0.11 \\
(-0.09-0.32)\end{array}$ & $\begin{array}{l}0.12 \\
(-0.07-0.31)\end{array}$ \\
\hline$>20 \%$ & $2.59 * * *$ & 0.74 & $0.23 *$ & 0.17 & 0.15 \\
\hline & $(1.51-4.47)$ & $(0.45-1.23)$ & $(0.03-0.43)$ & $(-0.03-0.37)$ & $(-0.05-0.34)$ \\
\hline \multicolumn{6}{|l|}{ Care coordination time } \\
\hline$\leq 8 \mathrm{~h}$ per week & & Ref & & Ref & Ref \\
\hline$>8$ h per week & & $0.54 * *$ & & $0.27 * *$ & $0.21 *$ \\
\hline & & $(0.37-0.81)$ & & $(0.10-0.43)$ & $(0.04-0.39)$ \\
\hline \multicolumn{6}{|l|}{$\begin{array}{l}\text { Provider satisfaction with } \\
\text { help received }\end{array}$} \\
\hline Number of observations & 402 & 400 & 400 & 400 & 400 \\
\hline R-squared & - & - & 0.10 & 0.12 & 0.16 \\
\hline Model & Logistic regression & Ordered logistic regression & \multicolumn{3}{|c|}{ Ordinary least square (OLS) regression } \\
\hline
\end{tabular}

$* * * p<0.001, * * p<0.01, * p<0.05$

${ }^{\dagger} 95 \%$ confidence intervals in parentheses

All regressions controlled for demographic, practice characteristics and medical center fixed effects (see Online Supplementary Appendix for full tables)

${ }^{\xi_{A}}$ 5-point single Likert-type agreement item where higher scores indicate greater provider satisfaction with help received in caring for high-risk patients. Effect size estimates refer to the odds of providers in the indicated category of the predictor variable reporting a higher level of satisfaction relative to the reference group

Derived as the average of four items rated on a 5-point Likert agreement scale with a higher score indicating greater reported stress from caring for highrisk patients. Effect size estimates refer to the change in reported stress of providers associated with a 1 SD increase in the predictor variable (if continuous) or the change in reported stress of providers in the indicated category of the predictor variable (if categorical) relative to the reference group

${ }^{\top}$ A 5-point single Likert-type agreement item where higher scores indicate greater provider satisfaction with help received in caring for high-risk patients. Scores were entered directly into the model

stress and care coordination time. In the former, the significance of the association between provider stress and proportion of HRPs disappeared after controlling for care coordination time (Table 2: Columns 3 and 4). In the latter, the magnitude of the association between care coordination time and provider stress diminished from 0.27 (Table 2, Column 4) to 0.21 points (Table 2, Column 5) when provider satisfaction with help received to care for HRPs is included.

Discussion. These results suggest that an increase in the proportion of HRPs in a PCP/nurse patient panel may impose a significant burden in terms of time spent coordinating care, which in turn was associated with increased stress. In addition, higher perceived satisfaction with help received in caring for HRPs was associated with less provider stress.

Consistent with our hypotheses, we found that the proportion of HRPs in PCP/nurse panels was associated with a greater amount of time spent coordinating care. Furthermore, perceived stress was associated with more time spent coordinating care and with less satisfaction with help received in caring for HRPs.

This is the first study to our knowledge that empirically links the proportion of HRPs in a $\mathrm{PCP} /$ nurse panel, care coordination of those patients, and associated stress. HRPs are usually managed by multiple providers, hence requiring extensive coordination of care among the relevant specialists and more time spent in care coordination. This is also consistent with the literature showing that patients with multimorbidity have a higher risk of care coordination problems. ${ }^{6-10}$

Consistent with the limited literature in this area, we found support for our hypothesis that increased care coordination demands on PCPs and staff in primary care may increase their level of stress. ${ }^{1}$ Forest et al. in 2010 reported increased provider satisfaction with improved care coordination experience in a pediatric primary care network. ${ }^{23} \mathrm{We}$ also found similar results in this study in the VHA PMCH setting. With a rise in the proportion of HRPs in primary care patient panels, care coordination demands will likely increase, and with it provider stress. Our findings suggest that providing adequate help for primary care staff may reduce the stress impact of increased care coordination. Help for primary care staff may be in the form of targeted initiatives such as intensive care management services or consultation on HRP care. Whatever form this takes, we found evidence to suggest that if providers are satisfied with the help and resources provided, especially if they perceive that the assistance is effective, they are more likely to experience less stress in caring for their HRPs. Early anecdotal evidence based on interviews with PCPs involved in the PACT Intensive Management demonstration 
suggests that having additional help may increase PCP confidence and the ability to care for HRPs.

Furthermore, we found no association between the proportion of HRPs and provider stress when we control for care coordination time and other relevant variables. This may suggest that provider stress is not affected by the proportion of HRPs that they manage; what seems to matter is how satisfied providers feel with available assistance.

As with all surveys, some limitations of this study include the self-reported cross-sectional nature of the data; hence, we can only report associations and are unable to make causal claims. While there is a possibility of self-report bias whereby respondents may over- or under-exaggerate their feelings of stress, we believe these subjective self-reports are a credible way of determining provider perceptions, a key goal of this study. In addition, our models explain about one-sixth of the variation in provider stress suggesting there are other important explanatory variables for which we did not account. It is also possible that our respondents are significantly different from non-respondents leading to response bias. However, we weighted for non-response, which attenuates the effects of such bias. It may also be difficult to generalize our study beyond the VHA since our sample has the unique advantage of an integrated delivery system structure with established PCMH teams that are associated with greater resources for care coordination and for vulnerable patients than typical smaller primary care practices. ${ }^{1}$ Also, our survey does not capture information about the specific kinds of help that providers receive in caring for their HRPs. Hence, further studies should investigate the resources that may alleviate provider stress in caring for HRPs.

Nevertheless, this study provides initial evidence on the challenge of HRP management. It sheds light on care coordination, a critical component of the PCMH model, and its relationship to provider stress in HRP management. Our findings suggest that adequate help for PCPs and nurses may reduce the stress they experience as they coordinate care for their HRPs, thus providing support for the need for targeted interventions to assist providers in these settings.

Funding and acknowledgements. The authors wish to thank Andrew Lanto, MA, for data management assistance at the VHA. We also thank Rosie Velasquez for help with the manuscript formatting; Jeremy Miles, $\mathrm{PhD}$, Bing Han, PhD, and Claude Setodji, PhD for statistical support at RAND. Finally, we wish to thank Donna Zulman, MD, MS, for her assistance with the literature and expertise on care coordination.

This work was undertaken as part of the VHA PACT Intensive Management (PIM) initiative, which pilots models of intensive primary care management for high-risk veterans. Funding for the PIM evaluation is provided by the VHA
Office of Patient Care Services (project no. XVA 65-054; Principal Investigator: LV Rubenstein).

An earlier version of the manuscript was presented as a poster at the AcademyHealth Annual Research Meeting, Boston, MA, in June 2016.

Corresponding Author: Adeyemi Okunogbe, MB.ChB, MSc; RAND Corporation, Santa Monica, CA, USA (e-mail: okunogbe@rand.org).

\section{Compliance with ethical standards:}

Conflict of interest: The authors declare that they have no conflicts of interest.

\section{REFERENCES}

1. Bodenheimer T. Coordinating care-a perilous journey through the health care system. New England Journal of Medicine 2008; 358(10): 1064.

2. Noël PH, Chris Frueh B, Larme AC, Pugh JA. Collaborative care needs and preferences of primary care patients with multimorbidity. Health Expectations 2005; 8(1): 54-63.

3. Skinner HG, Coffey R, Jones J, Heslin KC, Moy E. The effects of multiple chronic conditions on hospitalization costs and utilization for ambulatory care sensitive conditions in the United States: a nationally representative cross-sectional study. BMC Health Services Research 2016; 16(1): 1-8.

4. Zulman DM, Chee CP, Wagner TH, et al. Multimorbidity and healthcare utilisation among high-cost patients in the US Veterans Affairs Health Care System. BMJ open 2015; 5(4): e007771.

5. Powers BW, Chaguturu SK, Ferris TG. Optimizing high-risk care management. JAMA : the journal of the American Medical Association 2015; 313(8): 795-6.

6. Doessing A, Burau v. Care coordination of multimorbidity: a scoping study. Journal of Comorbidity 2015; 5(1): 15-28.

7. Zuchowski JL, Rose DE, Hamilton AB, et al. Challenges in referral communication between VHA primary care and specialty care. Journal of general internal medicine 2015; 30(3): 305-11.

8. Fried TR, Tinetti ME, Iannone L. Primary care clinicians' experiences with treatment decision making for older persons with multiple conditions. Archives of internal medicine 2011; 171(1): 75-80.

9. Maeng DD, Martsolf GR, Scanlon DP, Christianson JB. Care coordination for the chronically ill: understanding the patient's perspective. Health Services Research 2012; 47(5): 1960-79.

10. O'Malley A, Tynan A, Cohen GR, Kemper N, Davis MM, Lthpolicy PC. Coordination of care by primary care practices: strategies, lessons and implications. Research brief. 2009; 12.

11. Shanafelt TD, Hasan O, Dyrbye LN, et al. Changes in burnout and satisfaction with work-life balance in physicians and the general US working population between 2011 and 2014. Mayo Clinic proceedings; 2015: Elsevier; 2015. p. 1600-13.

12. Shanafelt TD, Sloan JA, Habermann TM. The well-being of physicians. The American Journal of Medicine 2003; 114(6): 513-9.

13. Wallace JE, Lemaire JB, Ghali WA. Physician wellness: a missing quality indicator. The Lancet 2009; 374(9702): 1714-21.

14. Henriksen $\mathbf{K}$, Battles JB, Marks ES. Advances in Patient Safety: from research to implementation (volume 4: programs, tools, and products). 2005. 
15. Schroeder SA. Physician supply and the US medical marketplace. Health Affairs 1992; 11(1): 235-43.

16. Landon BE, Reschovsky JD, Pham HH, Blumenthal D. Leaving medicine: the consequences of physician dissatisfaction. Medical Care 2006; 44(3): 234-42.

17. Sandy LG, Foster NE, Eisenberg JM. Challenges to generalism: views from the delivery system. Academic Medicine: Journal of the Association of American Medical Colleges 1995; 70(1 Suppl): S44-6.

18. AHRQ. Care Coordination. 2016. http://www.ahrq.gov/professionals/ prevention-chronic-care/improve/coordination/index.html (accessed March 3 2016).

19. McDonald K, Schultz E, Albin L, et al. Care Coordination Measure Atlas Version 4. Rockville: Agency for Healthcare Research and Quality; 2014.

20. McDonald K, Sundaram V, Bravada D, et al. Care coordination. Closing the quality gap: A critical analysis of quality improvement strategies. Technical Review 9 (Prepared by the Stanford University-UCSF Evidencebased Practice Center under contract 290-02-0017). Rockville: Agency for Healthcare Research and Quality; 2007.

21. Schultz EM, McDonald KM. What is care coordination? International Journal of Care Coordination 2014; 17(1-2): 5-24.

22. Parchman ML, Noël PH, Lee $\mathbf{S}$. Primary care attributes, health care system hassles, and chronic illness. Medical Care 2005; 43(11): 1123-9.

23. Forrest CB, Glade GB, Baker AE, Bocian A, von Schrader S, Starfield B. Coordination of specialty referrals and physician satisfaction with referral care. Archives of Pediatrics \& Adolescent Medicine 2000; 154(5): 499-506.

24. Werner RM, Canamucio A, Shea JA, True G. The medical home transformation in the Veterans Health Administration: an evaluation of early changes in primary care delivery. Health Services Research 2014; 49(4): 1329-47.

25. Helfrich CD, Dolan ED, Simonetti J, et al. Elements of team-based care in a patient-centered medical home are associated with lower burnout among VA primary care employees. Journal of General Internal Medicine 2014; 29(2): 659-66.

26. Meredith LS, Hackbarth NS, Darling J, et al. Emotional exhaustion in primary care during early implementation of the VA's medical home transformation: Patient-aligned Care Team (PACT). Medical Care 2015: 53(3): 253-60.

27. Helfrich CD, Li Y-F, Sharp ND, Sales AE. Organizational readiness to change assessment (ORCA): development of an instrument based on the Promoting Action on Research in Health Services (PARIHS) framework. Implementation Science 2009; 4(1): 38.

28. Reid RJ, Coleman K, Johnson EA, et al. The group health medical home at year two: cost savings, higher patient satisfaction, and less burnout for providers. Health Affairs 2010; 29(5): 835-43.

29. Robert J. Reid, Paul A. Fishman, Onchee Yu, Tyler R. Ross, James T Tufano, Michael P, Soman M, Larson EB. Patient-centered medical home demonstration: a prospective, quasi-experimental, before and after evaluation. Am J Manag Care 2009; 15(9): e71-e87. 